\title{
Index of Persons
}

A

Adamic Louis 170, 203, 293, 297

Adams Glenn 20

Adams Leonie 209

Adorno Theodor W. 164

Agar Herbert 33

Aiken Conrad 203, 213, 214

Ai Qing 216, 217, 221

Aldonow Marc 177

Aldridge Ira 248

Algren Nelson 51, 198

Anderson Marian 153

Anders Wtadystaw 151

Andrade Jorge Carrera 215, 220, 221

Andruszkiewicz Franciszek 16, 43

Andrzejewski Jerzy 11, 15, 16, 41, 68, 230

Apollinaire Guillaume 222

Appenszlak Jakub 14

Aragon Louis 41, 205

Arendt Hannah 7, 177, 185, 293

Aristophanes 44

Armstrong Louis 130

Arnold Matthew 174

Ash Sholem 198

Auden Wystan Hugh 7, 177, 180, 203, 206, 207, 209, 212, 213, 258, 286, 288, 291, 297

Auerbach Erich 201

Austin Jane 124

B

Babel Isaac 200

Balzac Honoré de 60, 63, 66, 77, 78, 102, 123, $129,172,189,191,278$

Barańczak Stanisław 2

Barker George 180

Barthé Richmond 166

Bataille Georges 181

Baudelaire Charles 224, 277, 278, 279

Beals Ralph A. 25

Bearden Romare 166

Bednarczuk Wincenty. See Putrament Jerzy

Bell Daniel 124, 139, 283, 286

Bellow Saul 199, 201

Benedict Ruth 33, 181, 200

Bénet Vincent 204

Benét William Rose 203

Benjamin Walter 222
Berdyaev Nicholas 274

Bergson Henri 171

Berlin Isaiah 201

Berlstein Alfred 25

Bernstein Leonard 130

Bernstein Samuel 33

Berryman John 180, 200, 201, 203

Bettelheim Bruno 178

Bian Zhilin 216

Bieńkowski Zbigniew 144, 145

Bigart Homer 19

Bishop Elizabeth 180, 199, 200, 203, 213

Blackmur Richard Palmer 201

Blake William 66, 174, 290

Bliss Lane Arthur 24

Błoński Jan 263

Boguszewska Helena 68

Bonnard Pierre 155

Borejsza Jerzy 21, 98, 294, 298

Borges Jorge Luis 200

Bór-Komorowski Tadeusz 151, 154

Borowski Tadeusz 64, 256, 260, 284

Boruta Kazys 227

Bosquet Alain 7, 213, 214

Bourdieu Pierre 261

Brahmer Mieczystaw 36

Brandys Kazimierz 67

Breza Tadeusz 10, 11, 12, 18, 19, 21, 30, 35

Breza Zofia 11, 12, 18, 30, 35

Bromfield Louis 190

Brooke Rupert 174

Brooks Van Wyck 203

Brown Earl 243

Browning Robert 174, 258, 281

Brown John 136, 166

Bruno Giordano 262

Buck Pearl S. 34, 199

Burns Robert 174, 216, 220

Butler Samuel 174

Byrnes James F. 108, 109, 113

Byron George 174

C

Caldwell Erskine Preston 34, 93, 142, 143, 185, 187, 188, 190

Campanella Tommaso 265

Campbell George 216, 221, 251 
Camus Albert 7, 185, 200

Canetti Elias 199

Capote Truman 199, 200, 201

Carberry Hugh Doston 216, 251

Carpenter Bogdana 2

Carrey Audrey 269, 276

Carroll Lewis 174

Cassirer Ernst 199

Cavaltanti Alberto 44

Cavanagh Clare 2, 258

Cayton Horace R., Jr. 244

Cesaire Aimé 230

Cézanne Paul 155, 191

Chagall Marc 34

Chaplin Charles 38, 44, 172

Chaucer Geoffrey 174

Chesterton Gilbert Keith 174

Chiang Kai-shek 114, 115

Chiaromonte Nicola 7, 185, 200, 293, 294

Chomski Leopold 133, 257

Chopin Frédéric 24, 42, 65, 240

Chudek Józef 16

Churchill Winston 24, 79, 105, 106

Ciardi John 176, 180, 200, 203, 279

Clair Réné 44

Clay Cassius Marcellus 149

Coleman Arthur Prudden 20, 36

Coleridge Samuel Taylor 174

Collier John 234, 235

Conrad Joseph (Józef Teodor Konrad Korzeniowski) 9, 71, 72, 78, 134, 144, 222, 233, 234, 236

Constable John 155

Cooper James Fenimore 63, 173, 226, 275

Copland Aaron 38

Corot Jean-Baptiste-Camille 155

Cortés Hernán de Monroy 230, 233

Courbet Gustave 66, 155

Cowley Malcolm 190, 193, 194

Cowper William 174

Crabbe George 174

Crane Hart 204, 298

Crowe John 180, 202

Cummings E.E. 180, 200

Czapski Józef 39

D

Dąbrowska Maria 36, 68

Dagover Lil 44

Daniderff Léo 303
Dante Alighieri 57, 79, 89, 259, 264

Darwin Charles 131, 260

David, King of Israel 249

Davidson David 99, 123, 176

Davie Donald 2

Davis Robert Gorham 201

Dęborylska Stanisława 11

Dedecius Karl 224

Defoe Daniel 77, 84, 124, 191

Degas Edgar 155, 165

De Kooning Willem 164

Delacroix Eugène 155

De Mille Cecil B. 44

Descartes René 79

DeVoto Bernard 176

Dewey Thomas E. 119

Dickens Charles 124, 196

Dickinson John 168

Disney Walt 166

Dłuski Ludwik 296

Dodson Owen V. 220

Donne John 258

Doolittle Hilda 180, 203, 286

Dos Passos John 63, 187, 190, 276

Dreiser Theodore 63, 187

Dreyer Carl Theodor 172

Drury Philip 17

Dryden John 174

Du Bois W.E.B. 243, 244

Dygat Stanistaw 18, 52, 67, 68

\section{E}

Eberhardt Richard 213

Ehrenburg llya 92, 185

Einstein Albert 34, 38, 45, 131, 132, 200, 257, 298

Eisenhower Dwight 183

El Greco 167

Eliot George 124

Eliot Thomas Stearns 7, 11, 15, 43, 67, 78, 80, 82, $85,86,173,174,186,203,204,205,206$, 207, 209, 210, 211, 212, 213, 217, 255, 258, 259, 284, 288, 290, 291, 297

Ellington Duke 130

Éluard Paul 205

Emerson Ralph Waldo 196

Erasmus of Rotterdam 206

Estreicher Karol 11

Eten Günther 133

Evans Luther 33 
Evans Walker 244

Evergood Philip 34

\section{$\mathrm{F}$}

Faggen Robert 2

Falconetti Renée Jeanne 44

Farrell James T. 177, 212

Fast Howard 191

Faulkner William 7, 32, 34, 37, 63, 80, 98, 136, $142,143,144,160,170,185,186,187,191$, 192, 193, 194, 217, 286, 288

Fiedler Arkady 230

Fiedler Leslie A. 201

Fielding Henry 77, 78, 124, 191, 278

Fitzgerald Ella 130

Fiut Aleksander 291

Flaubert Gustave 189, 191, 198

Fletcher John Gould 213

Foucault Michel 274

Frajlich-Zając Anna XV, 2, 148, 170

Franaszek Andrzej 42

Franczak Jerzy 259, 261, 262

Freud Sigmund 131, 210

Frohock Wilbur M. 93

Fromm Erich 200

Frost Robert 176, 177, 203, 206, 291, 297

G

Gajcy Tadeusz 72, 81

Gałczyński Konstanty Ildefons 67, 72

Galewicz Jan 25

Gauss Christian 15, 34, 156

Gericault Théodore 155

Gibbon Edward 43, 231, 232, 236, 298

Gide André 85, 86

Giedroyc Jerzy 39, 40, 148, 244

Gielgud Val Henry 150

Gogol Nikolai Vasilievich 192, 212

Gojawiczyńska Pola 18

Goldsmith Oliver 174

Gołębiowski Marek 128

Gombrowicz Witold 66, 68

Gomutka Władystaw 146

Goodman Benny 130

Gorczyńska Renata 48, 291

Gorky Arshile 164

Goya Francisco 155, 164

Gray Thomas 174

Greenberg Clement 166, 201

Greene Graham 200
Green William 134

Gronowicz Antoni 14

Grosz Wiktor 22

Grudzińska-Gross Irena 2

Grydzewski Mieczystaw 304

Grynberg Henryk XV, 70

Grzebieniowska Xenia 35

Guggenheim Peggy 16, 164, 165, 245, 246

Guillén Nicolás 220

$\mathrm{H}$

Haiman Mieczysław 149

Hardy Thomas 174

Hass Robert 2

Hauser Arnold 201

Hawthorne Nathaniel 196

Hegel Georg Wilhelm Friedrich 134

Heidegger Martin 274

Heller John 201

Heller Joseph 184, 199

Hellman Lillian 38

Hemar Marian 64

Hemingway Ernest 7, 34, 63, 97, 163, 184, 185, $187,188,189,190,191,195$

Herbert George 258

Hersch Jeanne 298

Hersey John 57, 180, 181, 182

Hertz Alexander 12, 26, 67, 92, 298

Hertz Paweł 23

Hesse Herman 200

Hillyer Robert 210

Hitler Adolf 54, 80, 211, 246

Hoffman Pawet 38

Holiday Billie 130

Hood Thomas 174

Hook Sidney 38, 177, 201, 294

Hoover Herbert C. 120, 296

Hopper Edward 166, 297

Horodyński Dominik 48

Horowitz David 110

Hughes Langston 38

Hugo Victor 205

Huxley Aldous 198, 222, 259, 275

I

Ingersoll Ralph 96, 177

Ingram Kenneth Everard 216, 221

Iribarne Louis 2

Iwaszkiewicz Anna 41

Iwaszkiewicz Jarosław 37, 41, 66, 214, 215, 240 
J

Jackowski Aleksander 93

Jacoby Annalee 114

Jakobson Roman 36

Jakubiak Katarzyna 215, 217

Janta-Połczyński Aleksander 14, 33, 39, 40, 42, $57,62,92$

Jarrell Randall 33, 101, 176, 180, 203, 258, 291

Jarzębski Jerzy 92, 134, 229, 254, 266

Jaspers Karl 7, 185

Jastrun Mieczysław 68

Jaworski Tadeusz 41

Jeffers Robinson 180, 200, 204, 206, 290

Jeleński Konstanty 293, 294

Jimenez Alberto Rojas 216

Johnson Helene 216, 251

Johnson James Weldon 247, 248

Jones Howard 111

Joyce James 186, 191

Judt Tony 103, 117

Jules Mervin 33

Julian the Apostate 79

Jung Carl Gustav 210

K

Kafka Franz 66, 76, 85, 86, 200

Karwowska Bożena 2

Kassern Tadeusz Zygfryd 25, 144, 145

Keats John 174, 229

Kennan George 100, 106, 113, 123

Kennedy John F. 154

Kenny Robert R. 34

Kipling Rudyard 174

Kisielewski Jerzy 52

Kisielewski Stefan 49, 67, 68, 144, 145, 146, 147

Kistler Marian 14

Klee Paul 165

Kline Franz 164

Kochanowski Jan 204

Koestler Arthur 183, 198, 200, 294, 295

Kołakowski Leszek 129

Komorowski Tadeusz. See Bór-Komorowski

Tadeusz

Kosciuszko Tadeusz 24, 168

Kossak-Szczucka Zofia 66

Kott Jan 67

Kowalska Anna 36, 41, 43, 44, 45

Kracauer Siegfried 201

Krasicki Ignacy 205

Krasiński Zygmunt 205
Krasner Lee 164

Kraus Werner 44

Kravchenko Victor 198

Kridl Manfred XV, 5, 14, 31, 32, 36, 53, 84, 89, 292, 294

Kridl Valkenier Elizabeth XV, 52

Kroński Tadeusz Juliusz 24, 41, 134, 151, 217, 232, 255, 256, 257, 276, 278, 284, 292, 298

Kruczkowski Leon 38

Krzywicka Irena 68, 144

Kunat Józefa 63

Kuryluk Karol 91, 93, 97, 98, 99, 145, 148, 180

Kuśniewicz Andrzej 10

\section{L}

Lanson Gustave 186

Łapiński Zdzisław XVI, 258

Lawrence D. H. (David Herbert) 195, 235, 238 , 275

Lawrence William 19

Layamon 174

Lechoń Jan 13, 51

Lec Stanistaw Jerzy 10

Lednicki Wacław 14

Leigh Hunt James Henry 174

Levine Madeline G. 2

Lewiński Thomas 149

Lewis Clive Staples 201

Lewis John 117, 118, 119

Lewis Sinclair 187

Liebman Joshua 198

Lilien Ernest 15

Lincoln Abraham 24, 112

Lindsay Vachel 204, 215, 220, 224, 250, 251

Lindtberg Leopold 166

Lippmann Walter 108, 113, 123, 126, 177

Lister Kazimierz 11

Locke Alaine 243

London Jack 63, 173, 196, 222

Longfellow Henry Wadsworth 63

Lorca Federico Garcia 215, 216, 220, 221, 224

Lorrain Claude 155

Lowell Robert 101, 176, 203, 206, 209, 258, 276, 278, 279, 288, 291, 292

Lukes Steven 262

M

Macdonald Dwight 38, 91, 94, 177, 179, 181, 285, 288, 294, 295, 298

MacLeish Archibald 33, 177, 257 
MacNeice Louis 180

Mailer Norman 43, 183, 184

Malinowski Władysław 14

Mann Thomas 38, 39, 99, 130, 132, 133, 134, 135, 144, 184, 200, 257, 287

Mao Zedong 114, 216

Marczyński Antoni 14

Maritain Jacques 78, 176

Markham Edwin 215, 220, 224, 250

Marquand John P. 190

Marshall George C. 109, 113, 114

Marx Karl 87

Masefield John 174

Masereel Frans 172

Masters Edgar Lee 85, 204

Matos Palés Luis. See Palés Matos Luis

Matthiessen Francis 0. 34

Matuszewski Ryszard 67, 94, 98, 186, 239, 247

McCarthy Mary 181, 293, 294

McCombs Charles F. 25

McCormick Anne O’Hare 110

McCullers Carson 199

McLuhan Marshall 125

McMarion Bruce 216, 221

Meader George 96, 107

Mellon Andrew William 210

Melville Herman 43, 196, 197, 198

Mencken Henry Louis 195, 201

Meredith George 174

Merleau-Ponty Maurice 7, 185, 200

Merrill John 200

Merton Thomas 199, 201, 285, 289

Merwin W.S. 214

Mickiewicz Adam 3, 5, 31, 32, 36, 37, 42, 48, 53, $61,81,82,205,259$

Mierzwa Stefan 20, 29

Mikołajczyk Stanisław 151

Miller Arthur 38

Miller Glenn 130

Miller Henry 7, 35, 177, 185, 194, 195, 196, 235, 288, 290, 297

Millet Jean-François 215, 250

Miłosz Andrzej 17

Mitosz Antoni VI

Mitosz Janina XIV, 10, 48

Miłosz Oscar 15, 41, 224, 290

Mitosz Weronika 220

Milton John 216

Mitchell Margaret 64

Mitchell Wesley Clair 34
Modzelewski Zygmunt 47

Monet Claude 155

Monk Thelonius 130

Monroe James 111

Montesquieu (Charles-Louis de Secondat) 284

Montezuma 233

Moore Marianne 203, 213, 214

Moravia Alberto 199

Morison Samuel Eliot 34

Mortkowicz-Olczak Hanna 67

Mounier Emmanuel 102

Mumford Lewis 29, 160

Myrdal Gunnar 198, 241, 243

$\mathrm{N}$

Nabokov Vladimir 199

Nałkowska Zofia 41, 42, 68

Nash Ogden 203

Natanson Jacek 144

Nathan Leonard 2

Neruda Pablo 27, 32, 37, 41, 177, 203, 215, 216, 217, 218, 220, 221, 281, 291, 298

Niemcewicz Julian Ursyn 168, 169

Nietzsche Friedrich 132, 134, 287

Norblin Stefan 35

Northrop F.S.C. 198

Norwid Cyprian Kamil 136, 205

Nowak-Jeziorański Jan 93

Nycz Ryszard XV, 82, 161, 194

0

Odets Clifforf 38

Ogrodziński Przemystaw 48

O’Keeffe Georgia 166

Olejniczak Józef XVI, 204, 205

O’Neill Eugene 199

Ordyński Ryszard 14

Orozco José Clemente 166

Ortega y Gasset José 201

Orwell George 177, 200

Osmańczyk Edmund Jan 33, 145

Ossowski Stanisław 38

P

Pageaux Daniel-Henri 148

Palés Matos Luis 216, 220, 251

Pankowski Marian 16

Panufnik Andrzej 35

Parandowski Jan 67

Parker Charlie 130 
Parker Dorothy 203

Parkman Francis 231, 232

Pepper Claude 105

Perón Juan 142

Perse Saint-John 45, 177, 203, 223, 291

Picasso Pablo 155, 165, 211

Pickett Virginia 52, 153, 155

Pinsky Robert 2

Pippin Horace 166

Piston Walter 34

Piwińska Marta 53, 90

Plato 88

Poe Edgar Allan 63, 173, 278, 297

Pollock Jackson 164

Pomian Grażyna 40

Pope Alexander 174

Porter James A. 243

Pound Ezra 200, 203, 204, 209, 211, 212, 215 , 265, 286

Poussin Nicolas 155

Pratt Fletcher 176

Préjean Albert 44

Proust Marcel 66, 85, 186, 191, 222, 229, 268

Pruszyński Ksawery 10, 14, 67, 68, 144

Przyboś Julian 10

Przybyszewski Stanisław 171

Pulaski Casimir 168

Pulitzer Joseph 128

Pushkin Alexander Sergeyevich 248

Putrament Jerzy 39, 41, 42, 46, 98, 144, 298

Pyle Ernie 179

$\mathrm{R}$

Rabelais François 77, 78, 197

Ransom John Crowe 180

Raphael (Raffaello Santi) 80

Raysman Wiktor 180

Reggio Godfrey 271

Rembrandt van Rijn 164, 167

Renoir Auguste 155

Rexroth Kenneth 2

Rimbaud Arthur 85, 222

Ripley Dwight 16

Rivera Diego 155, 166, 239

Robinson Edwin Arlington 204, 258

Roethke Theodore 213, 214, 217

Roosevelt Franklin Delano 28, 33, 104, 105, 108 , $112,115,118,123,124,198,288$

Rosenfeld Isaac 195, 196

Rosetti Dante Gabriel 174
Ross Alex 38

Rossellini Roberto 166

Rothko Mark 164

Rouault Georges 155

Roumain Jacques 216, 220, 251

Rousseau Henri (le Douanier) 155

Rożek Adolf 133, 257, 287

Różewicz Tadeusz 41, 42, 55, 75, 78, 85

Rudnicki Adolf 42

Rukeyser Muriel 213

Rusinek Michat 68

Ryńca Wtadystaw 40

Rzewuski Henryk 192

$\mathrm{S}$

Sacco Nicola 109

Said Edward W. 58

Salinger J.D. 199

Samuels Gertrude 20, 100

Sandauer Artur 144, 145

Sandburg Carl 204, 206, 216, 219, 224, 247, 249, 250, 259

Santayana George 199, 271

Saroyan William 36, 190

Sartre Jean-Paul 63, 64, 184, 187, 188, 189, 194, 199, 200

Schenker Alexander 2

Schiller Leon 36

Schlesinger Arthur, Jr. 177

Schulz Bruno 66

Schwartz Delmore 213

Scott Walter 174

Shakespeare William 44, 72, 124,131, 173, 204, 217, 221, 259, 272

Shallcross Bożena 2

Shapiro Karl 176, 180, 203, 205, 207, 209, 212, 258, 260, 276, 279, 286, 288

Shapley Harlow 34

Shaw George Bernard 195

Shaw Irwin 184

Shelley Percy Bysshe 174

Sholokhov Mikhail Aleksandrovich 222

Shostakovich Dmitri 38

Sienkiewicz Henryk 52

Silone Ignazio 7, 185

Simmel Georg 139

Sinclair Upton 63

Singer Isaac Bashevis 199

Siquerios David Alfaro 166, 239

Skarżyński Tadeusz 35 
Sławiński Janusz 294

Stonimski Antoni 10, 11, 47

Stowacki Juliusz 61, 205, 259

Smith William Gardner 43, 179, 183

Socrates 271

Sommers, musician 161, 181, 182

Spellman Francis Joseph, Cardinal 154

Stalin Joseph Vissarionovich 107, 115, 150, 152, 288

Steinbeck John 34, 36, 93, 98, 185, 187, 190

Stendhal (Marie-Henri Beyle) 196, 222

Sternberg Fritz 96, 99, 123

Stevens Wallace 7, 180, 200, 201, 202, 214, 222, 286

Stone Isidor F. 20, 123, 177

Storm Jameson Margaret 11, 293, 294

Stowe Harriet Beecher 239

Straszewicz Czestaw 285

St. Vincent Millay Edna 180

Supervielle Jules 41

Suszkiewicz Metody 15

Swanson Gloria 44

Swedenborg Emmanuel 290

Święcicki Ignacy 14

Swift Jonathan XIII, 25, 77, 78, 124, 255, 257, 258, 263

Swinburne Charles Algernoon 174

Syker Gerald 177

Sym Igo 61

Szmaglewska Seweryna 18, 53, 76

Szpilman Władysław 300

Szymanowski Karol 24

\section{T}

Taft Robert A. 119, 120

Taine Hippolyte 157

Tarbell Ida M. 128

Tate Allen 177, 180, 200, 209

Telakowska Wanda 5, 34, 35, 36, 41, 80, 194, 234, 235, 238, 275

Tennyson Alfred 174

Terlecki Tymon 11

Themerson Franciszka 11

Themerson Stefan 11

Thomas Dylan 180, 201, 203

Thompson Dorothy 96, 100, 177

Thoreau Henry David 196

Tian Jian 217

Tintoretto Jacopo 80
Tolstoy Lev Nikolayevich 60, 78, 85, 86, 87, 196, 272

Topolski Feliks 11

Toynbee Arnold J. 199, 200

Traherne Thomas 173

Trilling Lionel 201

Truman Harry S. XII, 6, 24, 79, 95, 100, 105, 106, $107,108,109,110,111,112,113,114,115$, $116,117,119,120,121,288$

Turowicz Jerzy 41

Twain Mark 63, 173, 196

Tyszkiewicz Elżbieta 35

Tyszkiewicz-tącki Janusz Maria 35

$\mathrm{U}$

Undset Sigrid 191

Unkiewicz Tadeusz 32

Untermeyer Louis 203

V

Valdes Ildefonso Pereda 216

Valee Lillian 2

Vandenberg Arthur H. 113

Van Doren Mark 203

Vanzetti Bartolomeo 109

Veidt Conrad 44

Vendler Helen 2

Viereck Peter 213, 214

Voltaire (François-Marie Arouet) 172

W

Walker Margaret 216, 251

Wallace Henry A. 28, 33, 38, 91, 93, 95, 100, 105, $108,117,295$

Walska Ganna 35

Wańkowicz Melchior 285, 293

Warren Robert Penn 180, 199

Watteau Jean-Antoine 270

Ważyk Adam 32, 42, 222

Węgierski Tomasz Kajetan 168, 169

Weil Simone 7, 179, 185, 293, 298

Wells Herbert George 99, 109, 130, 131, 132, 133, 135, 259, 298

Welscott Glenway 177

Wen Yiduo 216

West Dorothy 199

White Theodore H. 114

White Walter 244

White William L. 179 
Whitman Walt 82, 97, 173, 196, 206, 215, 216,

220, 224, 249, 250, 295, 297

Wiene Robert 44

Wierbłowski Stefan 47

Wierzyński Kazimierz 51

Wilde Oscar 7, 174, 208

Wilder Thornton 16, 45, 63, 177, 198, 291

Williams Tennessee 170, 199

Williams William Carlos 231, 232, 286, 297, 298

Wilmot John 174

Wilson Edmund 37, 176, 177, 186, 187

Wilson Mira Bigelow 32

Winiewicz Józef 13, 25, 26, 48

Wionczek Mieczystaw 144, 146

Witkiewicz Stanisław Ignacy (Witkacy) 40, 65, 66

Wittlin Halina 68

Wittlin Józef 14, 40, 122, 129, 200

Wnukowa Józefa 33, 68

Woolf Virginia 201

Wordsworth William 174

Wreszin Michael 39

Wright Bruce McMarion 216, 221, 251

Wright Richard 191

Wróbel Jack 120

Wyeth Andrew 166

Wyka Kazimierz 98, 204

Wyler William 123

Wylie Philip 199

Wyspiański Stanisław 61

Y

Yarmolinsky Avrahm 25, 26

Yu Min-Chan 216, 219

Z

Zagajewski Adam 2

Zagórski Jerzy 10, 144, 145

Zaleski Marek 298

Zang Kejia 217, 220

Żarnower Teresa 17, 260

Żeromski Stefan 50

Zielonko Jane 276, 293

Ztotowska Mira 14

Ztotowski Ignacy 25

Znaniecki Florian 136

Zola Émile 198

Zukofsky Louis 201

Żyranik Bogdan 230 